\title{
CREACIÓN DE ENTORNOS PERSONALES DE APRENDIZAJE COMO RECURSO PARA LA FORMACIÓN. EL PROYECTO DIPRO 2.0
}

\section{CREATING PERSONAL LEARNING ENVIRONMENTS AS A RESOURCE FOR TRAINING. THE DIPRO 2.0 PROJECT}

\author{
Julio Cabero Almenara \\ cabero@us.es \\ Universidad de Sevilla
}

\section{RESUMEN}

A través del presente artículo se ofrece una primera aproximación al concepto de PLE en lo que corresponde a los componentes fundamental, herramientas que lo configuran, así como diferentes perspectivas o marcos de diseño instruccional desde lo que poder enmarcarlos para su aplicación didáctica en contextos de formación de enseñanza superior. La última parte del mismo, está destinada a la exposición del proyecto Dipro 2.0 desarrollado con el objetivo principal de construir un entorno personal de aprendizaje que sirviese como herramienta tecnológica-didáctica y curricular desde el que formar al profesorado universitario, así como para la formación de los estudiantes en temáticas vinculadas con las tecnologías educativas.

Palabras clave: Entornos personales de aprendizaje, aprendizaje autorregulado, formación del profesorado, educación superior, web 2.0.

\begin{abstract}
Through this article provides a first approximation to the concept of PLE, which corresponds to the key components, tools that make it up, and different perspectives and instructional design frameworks from which to frame them for use in training contexts didactic higher education. The last part of it, is intended to exposure Dipro 2.0 project developed with the primary objective of building a personal learning environment that would serve as a technological-curricular teaching and from which university to train teachers and to the formation of students on topics related to educational technology.
\end{abstract}

Keywords: Personal learning environments, self-regulated learning, teacher training, higher education, web 2.0. 


\section{INTRODUCCIÓN.}

Como ha llamado la atención el Informe Horizonte denominado "Perspectivas Tecnológicas. Educación Superior en Iberoamérica 2012-2017" (Durall y otros, 2012), diferentes tecnologías van a llamar a las puertas de las Instituciones educativas universitarias iberoamericanas para su implantación en un horizonte cercano; así a un horizonte de implantación de un año o menos, las que más impactarían serían las aplicaciones móviles, la computación en nube, los contenidos abiertos, y los entornos colaborativos; y a un horizonte de dos a tres años, las tabletas, el aprendizaje basado en juegos, los entornos personales de aprendizaje y la geolocalización.

Al análisis de esta tecnología es a la que nos vamos a centrar en el presente artículo, analizando diferentes aspectos, que van desde qué podemos llegar a entender por ellos, qué posibilidades nos ofrecen para la formación, o qué diferencias podemos establecer con otras tecnologías utilizadas en la formación virtual como los LMS ("Learning Managemnet System"). Para finalizar, presentaremos los entornos producidos por nosotros enmarcado en un proyecto de investigación financiado que perseguía, como objetivo principal, elaborar un PLE.

\section{LOS ENTORNOS PERSONALES DE APRENDIZAJE.}

Hablar de PLE, supone, en primer lugar, referirnos a diferentes aspectos relacionados con cómo aprenden los alumnos en la sociedad del conocimiento, con cómo los conocimientos se hacen más líquidos y menos rígidos -utilizando el planteamiento de Bauman (2007)-, y con cómo las instituciones formativas regladas dejan de ser las únicas estancias y los únicos mecanismos donde las personas adquieren conocimientos, competencias y capacidades.

Ahondando en el primero de los aspectos, y de acuerdo con Starkey (2011), podemos decir que el aprendizaje en la era digital se produce no reproduciendo contenidos, sino abordándolo desde nuevas perspectivas, como son: haciendo actividades sobre ellos, pensado sobre las conexiones que con ellos podemos establecer, criticando y evaluando la información aportada, y creando y compartiendo conocimientos con otras personas. Frente a la idea de percibir el conocimiento como algo estático que reside en nuestra mente, desde las nuevas visiones sobre el aprendizaje, se asume que el mismo es algo fluido y que es construido y recreado mediante una participación activa de la persona. Como nos sugiere Downes (2007), se aprende más a través de la interacción que de la simple presentación de hechos.

Por otra parte, no debemos olvidar que la propia dinamicidad de la sociedad del conocimiento (Barroso y Cabero, 2013), hace que la durabilidad de los conocimientos y la información que se genera sea menor que como ocurría en la sociedad postindustrial, de ahí que se deban buscar mecanismos para que las personas puedan reconstruir su bagaje 
de conocimiento con los mínimos esfuerzos posibles, y ahí las tecnologías que están emanando de la web 2.0 y los "social media" se están convirtiendo en poderosas herramientas de ayuda. Situaciones como las que estamos tratando están repercutiendo para que las tradicionales instituciones de formación (escuelas, universidades,...), es decir las instituciones "formales", no sean las únicas estancias y contextos donde adquiramos los conocimientos, y nuestras posibilidades se amplíen con los contextos no formales e informales, y con la combinación de lo presencial y lo virtual (Attwell, 2007; Banyard y Underwood, 2008; Cedefop, 2008; Dabbagh y Kitsantas, 2011).

En este contexto de cambios y transformaciones es donde surgen los Entornos Personales de Aprendizaje (Personal Learning Environments) o PLE como un modelo emergente de formación virtual relacionado con el aprendizaje autónomo de los estudiantes, como dirá Adell $(2013,274)$ :

Aspectos como los comentados anteriormente, llevan a señalar a Dabbagh y Kitsantas $(2012,4)$, que "un PLE es una construcción nueva en la literatura e-learning que se basa en los medios de comunicación social y va ganando terreno en el e-aprendizaje como una plataforma eficaz para el aprendizaje del estudiante". O como han sugerido Barroso, Cabero y Vázquez (2012), nos ofrecen "la posibilidad de incorporar las herramientas de la Web 2.0 y las redes sociales desde una nueva perspectiva formativa, haciendo que la persona adquiera nuevas formas de desenvolverse en el contexto formativo virtual."

Ahora bien, ¿qué podemos entender por entornos personales de aprendizaje?

En este aspecto, Cabero y otros (2011) han agrupado las definiciones que se han ofrecido sobre el concepto, las cuáles se pueden agrupar en dos grandes tendencias: tecnológicas/instrumentales y pedagógicas/educativas. Con la primera, se refiere a un conjunto de herramientas de aprendizaje, servicios y artefactos, recogidos de diversos contextos y entornos para que sean utilizados por los estudiantes. Mientras que en la segunda, se hace hincapié en el componente de la aplicación educativa, desde la cual pueden ser considerados como sistemas que ayudan a los profesores y a los estudiantes para que establezcan sus metas de aprendizaje y los mecanismos por los cuales quieren llegar a él.

En la primera de las posiciones se incluyen diferentes autores (Fiedler y Pata, 2009; Amine, 2009; Reig, 2009; y Henri y otros, 2008), que los entienden como "una colección autodefinida de recursos, servicios, herramientas y dispositivos que sirven para que los profesores y alumnos puedan conformar sus redes personales para el aprendizaje y el conocimiento." (Cabero y otros, 2011).

Desde la segunda de las perspectivas, en la cual se encuentran autores como Casquero y otros (2010), Attwell (2007) o Adell y Castañeda (2010), se pone el acento en su aplicación educativa y en su consideración como una nueva metodología educativa. $Y$ desde esta perspectiva, y como hemos aludido anteriormente, podríamos decir que el concepto de PLE 
incluye la integración de elementos de la formación tanto formal como informal y no formal en una experiencia única de aprendizaje, así como el uso de redes sociales que pueden cruzar las fronteras institucionales y la utilización de protocolos de red (peer-topeer, servicios web, sindicación de contenidos...).

Desde esta segunda posición, el esfuerzo no se sitúa en la incorporación de diferentes tipos de herramientas, sino más bien en las diferentes decisiones que el alumno adopta para personalizar su aprendizaje y autorregularlo.

Lógicamente, el tomar la decisión de centrarnos en una posición u otra, tendrá claras repercusiones, pues una se encuentra más centrada en la herramienta y otra en la pedagogía (Ehiyazaryan-White, 2012); una, nos llevará a reflexionar sobre la accesibilidad de las tecnologías, su potencialidad y facilidad de manejo, o a su combinatoria; por el contrario, la otra nos llevará a reflexionar sobre el aprendizaje autorregulado, o las formas en las cuales los estudiantes se pueden acercar a la realización de las tareas

Al mismo tiempo, como nos llaman la atención diferentes autores (Wilsonn, 2008; Adell y Castañeda, 2010; Uztarroz, 2010; Väljataga, Pata, y Tammets, 2011), asumir la segunda de las posiciones es reflexionar respecto a dónde se aprende, dónde se obtiene la información, dónde se relaciona el alumno para el aprendizaje, y dónde se construye la información.

En cuanto a los componentes fundamentales que pueden configurar un PLE, la estructura que plantean Castañeda y Adell (2013) resulta significativa, en tanto en cuanto establecen sus partes a través de los siguientes elementos:

a. "Herramientas y estrategias de lectura: las fuentes de información a las que accedo que me ofrecen dicha información en forma de objeto o artefacto (mediatecas);

b. Herramientas y estrategias de reflexión: los entornos o servicios en los que puedo transformar la información (sitios donde escribo, comento, analizo, recreo, publico), $y$

c. Herramientas y estrategias de relación: entornos donde me relaciono con otras personas de/con las que aprendo". 


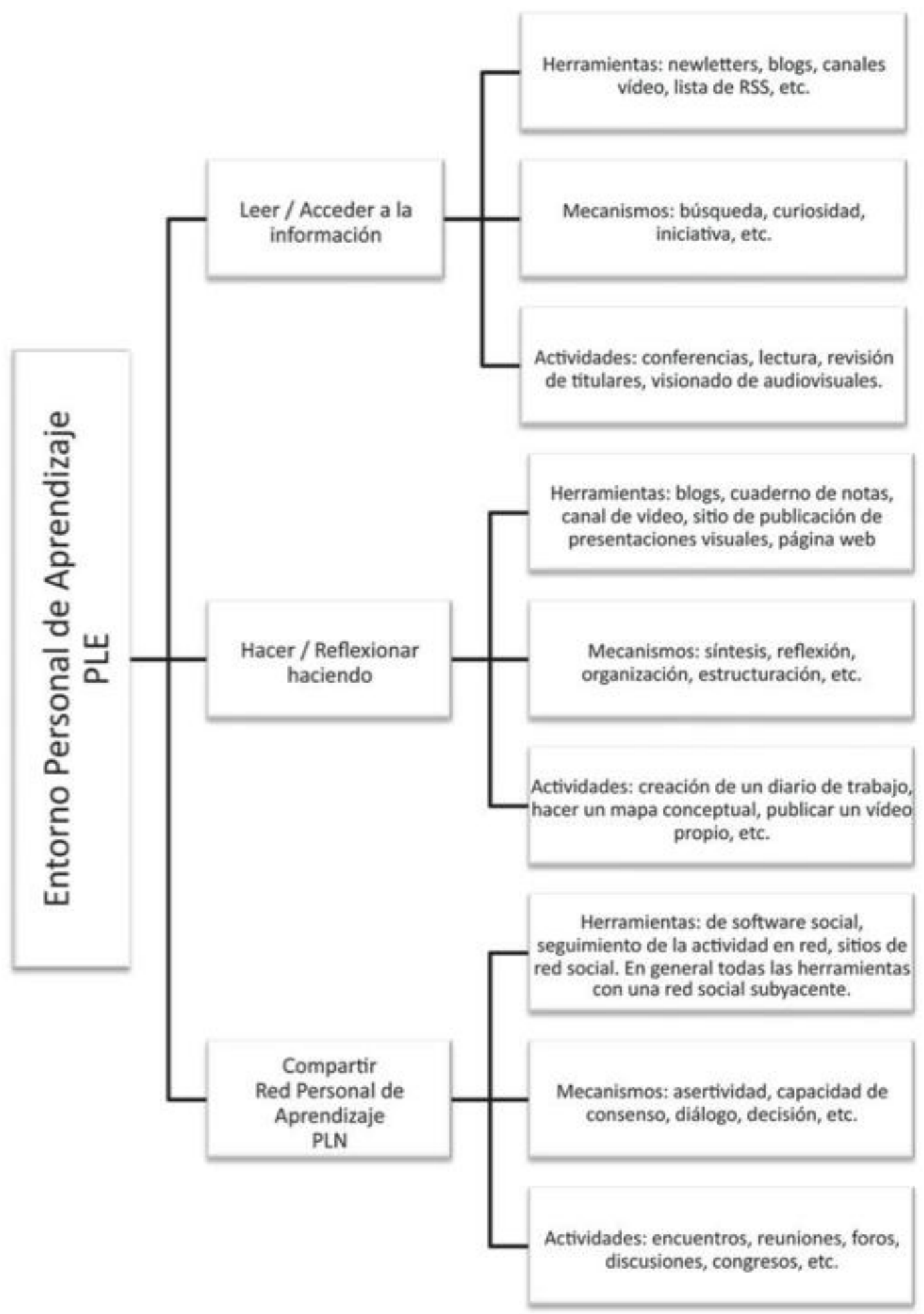

Figura 1. Componentes de un PLE. Fuente: Castañeda y Adell $(2013,20)$.

Todos estos elementos, componentes y herramientas repercuten en las transformaciones en los roles a desempeñar por los profesores y los estudiantes en el proceso formativo; los primeros, dejan de ser los únicos depositarios de la información, y se convierten más en diseñadores de situaciones medidas de aprendizaje, y los segundos, adquieren un papel más activo en su proceso formativo. En otro trabajo, Llorente (2013) apuntaba como los profesores podían guiar y orientar a los estudiantes en el uso de los medios sociales en los 
diferentes niveles de estructura que Dabbagh y Reo (2011) y Kitsantas y Dabbagh (2010) establecía para incorporar la creación del propio PLE por parte de los estudiantes como ayuda y soporte en lo que a las habilidades de autorregulación se refiere, y que se estructuraban en: (1) la gestión de la información personal, (2) la interacción social y la colaboración, y (3) la agregación de información y gestión.

\begin{tabular}{|c|c|c|c|}
\hline & $\begin{array}{l}\text { (Nivel 1) gestión de la } \\
\text { información personal } \rightarrow\end{array}$ & $\begin{array}{l}\text { (Nivel 2) La interacción } \\
\text { social y la colaboración } \\
\rightarrow\end{array}$ & $\begin{array}{l}\text { (Nivel 3) La agregación } \\
\text { de información y gestión } \\
\rightarrow\end{array}$ \\
\hline Blogs & $\begin{array}{l}\text { El profesor anima a los } \\
\text { estudiantes a utilizar el } \\
\text { blog como un diario } \\
\text { privado para establecer } \\
\text { metas de aprendizaje y } \\
\text { establecer la } \\
\text { planificación del curso y } \\
\text { las tareas. }\end{array}$ & $\begin{array}{l}\text { El profesor fomenta en } \\
\text { los estudiantes la } \\
\text { activación de la función } \\
\text { de comentarios en el blog } \\
\text { para permitir la } \\
\text { retroalimentación de los } \\
\text { compañeros y del } \\
\text { profesor. }\end{array}$ & $\begin{array}{l}\text { El docente muestra } \\
\text { cómo configurar un blog } \\
\text { para incorporar } \\
\text { contenido adicional y } \\
\text { cómo añadir en el blog } \\
\text { servicios de agregación } \\
\text { RSS. }\end{array}$ \\
\hline Wikis & $\begin{array}{l}\text { El profesor anima a los } \\
\text { estudiantes a usar las } \\
\text { wikis como un espacio } \\
\text { personal para la gestión } \\
\text { y organización del } \\
\text { contenido. }\end{array}$ & $\begin{array}{l}\text { El docente anima a los } \\
\text { estudiantes a activar las } \\
\text { funciones de edición } \\
\text { colaborativa } \\
\text { comentarios para generar } \\
\text { retroalimentación. }\end{array}$ & $\begin{array}{l}\text { El docente muestra } \\
\text { cómo ver el historial de } \\
\text { un wiki para promover la } \\
\text { auto-evaluación de su } \\
\text { aprendizaje a través del } \\
\text { tiempo. }\end{array}$ \\
\hline Calendario de Google & $\begin{array}{l}\text { Instructores animan a los } \\
\text { estudiantes a utilizarlo } \\
\text { como } \\
\text { personal. }\end{array}$ & $\begin{array}{l}\text { Instructor anima a los } \\
\text { estudiantes a usar las } \\
\text { funciones colaborativas y } \\
\text { de uso compartido del } \\
\text { calendario para permitir } \\
\text { la retroalimentación y } \\
\text { colaboración en el } \\
\text { desarrollo de las tareas } \\
\text { del curso. }\end{array}$ & $\begin{array}{l}\text { El profesor muestra } \\
\text { cómo archivar } \\
\text { calendarios personales y } \\
\text { de grupo para promover } \\
\text { la auto-valoración con } \\
\text { respecto a la } \\
\text { planificación y gestión de } \\
\text { tiempo. }\end{array}$ \\
\hline YouTube o Flickr & $\begin{array}{l}\text { El docente anima a los } \\
\text { estudiantes a usar Flickr } \\
\text { o YouTube para crear un } \\
\text { archivo personal de } \\
\text { medios relacionado con } \\
\text { el contenido del curso. }\end{array}$ & $\begin{array}{l}\text { El profesor anima a los } \\
\text { estudiantes a utilizar el } \\
\text { uso compartido de los } \\
\text { archivos de medios y a } \\
\text { unirse a otras creadas por } \\
\text { sus compañeros. }\end{array}$ & $\begin{array}{l}\text { El docente muestra } \\
\text { cómo agregar medios y } \\
\text { archivos para refinar su } \\
\text { archivo personal. }\end{array}$ \\
\hline Redes Sociales & $\begin{array}{l}\text { El profesor anima a los } \\
\text { estudiantes a crear un } \\
\text { perfil académico y } \\
\text { profesional en Linkedın. }\end{array}$ & $\begin{array}{l}\text { El docente anima a los } \\
\text { estudiantes a conectarse } \\
\text { con las comunidades } \\
\text { sociales relacionados con } \\
\text { sus objetivos } \\
\text { profesionales. }\end{array}$ & $\begin{array}{l}\text { Instructor solicita que los } \\
\text { estudiantes se } \\
\text { comprometan en su } \\
\text { propia auto-reflexión } \\
\text { para conseguir el } \\
\text { objetivo de reestructurar } \\
\text { su perfil y su presencia } \\
\text { social. }\end{array}$ \\
\hline Marcadores Sociales & $\begin{array}{l}\text { El docente anima a los } \\
\text { estudiantes a utilizar una } \\
\text { herramienta de } \\
\text { marcadores sociales (por } \\
\text { ejemplo, Delicious) para }\end{array}$ & $\begin{array}{l}\text { El profesor anima a los } \\
\text { estudiantes a colaborar } \\
\text { con otros compañeros de } \\
\text { clase y crear una lista } \\
\text { compartida }\end{array}$ & $\begin{array}{l}\text { El profesor pide a los } \\
\text { estudiantes } \\
\text { reflexionen sobre sus } \\
\text { marcadores personales y } \\
\text { de grupo para mejorar }\end{array}$ \\
\hline
\end{tabular}




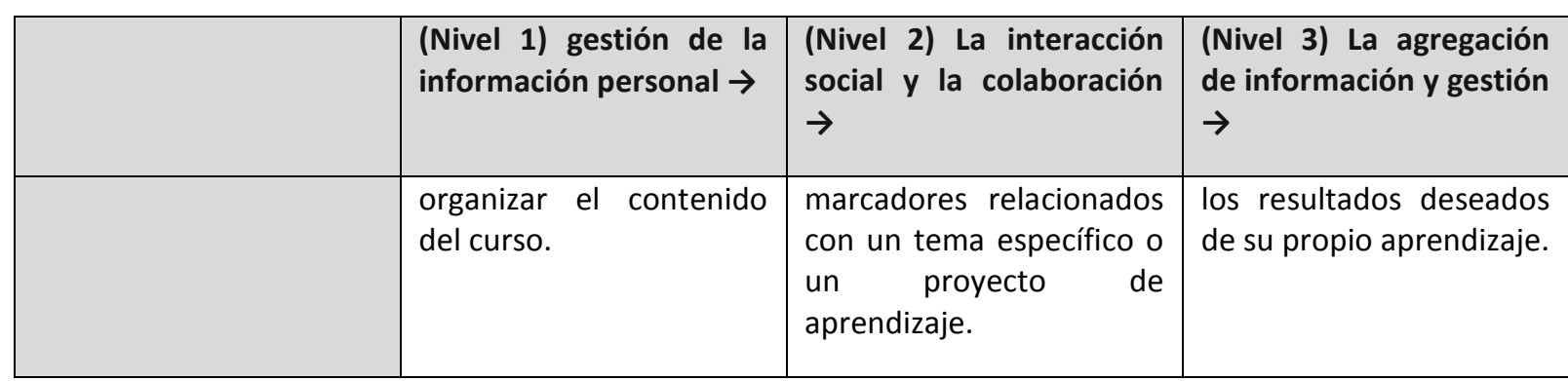

Tabla 1. Marco teórico para el uso de los medios sociales como soporte del aprendizaje autorregulado en entornos de aprendizaje personales (PLE). Fuente: Elaboración propia a partir de las aportaciones de Dabbagh y Kitsantas $(2012,7)$

Por otro lado, de acuerdo con Mott (2010), podemos se pueden apuntar una serie de fortalezas y debilidades que presentan los PLE para su incorporación a la práctica educativa, que sintetizamos en la siguiente tabla.

\begin{tabular}{|c|c|}
\hline \multicolumn{2}{|c|}{ PLE } \\
\hline Fortalezas & Debilidades \\
\hline $\begin{array}{l}\text { Variedad y funcionalidad casi ilimitada de } \\
\text { herramientas personalizables y adaptables } \\
\text { con múltiples configuraciones y variaciones. } \\
\text { Barato - a menudo desarrollado con } \\
\text { herramientas de software libre. } \\
\text { No hay límites de tiempo impuestos: } \\
\text { permanece en "ON" antes, durante y después } \\
\text { de la matriculación. } \\
\text { Abierto a la interacción, el intercambio y la } \\
\text { conexión sin tener en cuenta la inscripción } \\
\text { oficial en programas o cursos privados o } \\
\text { institucionales. } \\
\text { Centrado en los estudiantes (cada estudiante } \\
\text { selecciona y utiliza las herramientas que } \\
\text { precisa para sus necesidades y circunstancias } \\
\text { particulares). } \\
\text { Los contenidos de aprendizaje y las } \\
\text { conversaciones son compilables a través de } \\
\text { tecnologías simples como RSS. }\end{array}$ & $\begin{array}{l}\text { Complejo y difícil de crear para los } \\
\text { estudiantes y profesores sin experiencia } \\
\text { Problemas potenciales para la seguridad de } \\
\text { los datos. } \\
\text { Limitado control institucional sobre los datos. } \\
\text { No cuentan con servicios de apoyo técnico } \\
\text { que permitan predecir o resolver los } \\
\text { problemas que surjan en las aplicaciones } \\
\text { web, pudiendo incluso llegar a desaparecer. } \\
\text { Carece de gestión centralizada, los usuarios } \\
\text { se agregan a las listas de los grupos de } \\
\text { interés. } \\
\text { El que pueda proporcionar el soporte } \\
\text { adecuado para integrar variedad de } \\
\text { herramientas web e incorporarse a los } \\
\text { sistemas institucionales, resulta complejo y } \\
\text { costoso. }\end{array}$ \\
\hline
\end{tabular}

Tabla 2. Fortaleza y debilidades de los PLE (Fuente: Mott, 2010.)

Para un grupo de autores (Taraghi y otros, 2009; Brown, 2010; Ampudia y Trinidad, 2012), para entender bien la significación que tiene un PLE puede ser de gran ayuda establecer diferencias significativas respecto a los LMS, ya que estos últimos, más que favorecer la realización de actividades de formación innovadoras lo que están propiciando son acciones formativas tradicionales, y lo único que han supuesto es trasladar y reproducir las acciones formativas presenciales a contextos virtuales; y más que favorecer acciones formativas innovadoras, están propiciando acciones formativas conservadoras y tradicionales, sirviendo en realidad su fuerte penetración, no tanto desde un punto de vista formativo, sino más bien para el control institucional de los estudiantes. Todo ello, sin olvidarnos que los profesores no suelen utilizar todas las funciones que nos ofrecen los LMS (Sclater, 2008). 
Como señalan Dabbagh y Kitsantas $(2012,4)$ "Los LMS siempre han estado bajo el control de la institución, sus profesores y administradores, dejando poco espacio a los estudiantes para administrar y mantener un ambiente de aprendizaje, que facilite el desarrollo de sus propias actividades de aprendizaje, así como conexiones con sus pares y las redes sociales". De todas formas, y como posteriormente veremos, podemos crear entornos que combinen las dos tecnologías apuntadas.

No obstante, como matizan algunos autores (Casquero y otros., 2010; Marín, 2011) no es necesario contraponer PLE y LMS, y perfectamente pueden combinarse para incorporarlos en acciones formativas formales con el objeto de crear un verdadero ecosistema de formación virtual, que permita, tanto el acceso a los contenidos del curso, como el acceso libre y flexible a otros escenarios de comunicación e interacción, pertenezcan o no al contexto reglado de formación de la institución.

Bajo esta perspectiva hemos organizado nuestro entorno Dipro 2.0, configurándolo como la combinación de los dos componente, para dar lugar a una nueva realidad que podemos denominar como iPLE o Pleanring; que como hemos señalado procedería de la combinación de las posibilidades de la formación virtual (e-learning) y la flexibilidad de los PLE para alcanzar en su combinación nuevos escenarios de formación virtual, que permitan a los estudiantes tanto la interacción con los contenidos a través del LMS, y el buscar y/o filtrar información, organizarla y generar nuevos contenidos y compartirlos con su comunidad, a través de las diferentes herramientas que compongan su PLE.

Para finalizar los comentarios realizados respecto a los PLE, se presentan algunos aspectos que indica Rodríguez $(2013,10)$ y que creemos puede servirnos de síntesis de los aspectos comentados:

- Hablar de PLE, es referirnos a innovación educativa, a nuevas formas de aprender en la sociedad del conocimiento, y a asumir que la formación del ciudadano del futuro se movilizará dentro de lo denominado como formación formal, no formal e informal.

- Hablar de PLE, es no olvidarnos que siempre han existido personas que en su relación nos han ayudado en la construcción de nuestro conocimiento. La diferencia en la actualidad es que las herramientas de comunicación de la web 2.0, fundamentalmente las redes sociales, facilitan enormemente la nueva construcción del conocimiento.

- Hablar de PLE, es referirnos a una tecnología que bien organizada y estructurada, desde los momentos iniciales, nos puede acompañar a lo largo de nuestro proceso formativo, independientemente de su institucionalización o no.

- Hablar de PLE, es referirnos más a los procesos de cómo aprenden los estudiantes, las personas, que a cómo enseñan los profesores. Es por tanto una tecnología referida más a la construcción significativa y mediada del conocimiento, que a la forma de ofrecer información y contenidos a los estudiantes.

- Hablar de PLE, es referirnos directamente a cómo aprenden los alumnos en la Sociedad del Conocimiento.

- Hablar de PLE, es pasar de modelos de enseñanza centrados en el profesor a modelos centrados en el estudiante. 
- Y por último hablar de PLE, es contemplar directamente que vamos a trabajar con modelos donde lo importante no es el escuchar sino más el conectar; y no es para acceder a la información sino también, y es lo verdaderamente importante desde nuestro punto de vista, para producirla.

Realizados estos comentarios previos, en los siguientes apartados del presente artículo se presentan las características generales de un PLE, elaboradas a la luz del proyecto financiado por el Ministerio de Ciencia e Innovación del Gobierno Español denominado "Diseño, producción y evaluación de un entorno de aprendizaje 2.0 para la capacitación del profesorado universitario en la utilización educativa de las Tecnologías de la Información y la Comunicación" (Dipro 2.0 EDU2009-08893).

\section{ALGUNOS APUNTES SOBRE EL PROYECTO.}

El proyecto citado perseguía los siguientes cuatro objetivos generales:

1. Elaborar temáticas básicas, de forma consensuada, entre diferentes profesionales del ámbito de la Tecnología de la Educación sobre las áreas más significativas en las cuales debe capacitarse al profesorado universitario para el manejo didáctico de las TIC.

2. Crear un entorno formativo telemático bajo la arquitectura web 2.0, destinado a la formación del profesorado universitario en la adquisición de diferentes capacidades y competencias para la utilización e inserción de las TIC en su actividad profesional.

3. Validar el entorno formativo telemático, tanto en lo que se refiere a la propuesta de estructurar los contenidos, como a las diversas herramientas de comunicación (blog, wikis,...) creadas.

4. Configurar una comunidad virtual de profesorado universitario preocupado por la utilización educativa de las TIC, y por la formación del profesorado para el uso de las TIC.

En el presenta artículo se hará referencia a los tres primeros objetivos indicados, ya que los que se refieren a la Comunidad Virtual se encuentra en la siguiente dirección web: http://dipro20.ning.com/, a través de la cual el lector interesado puede solicitar su presencia en la misma.

En nuestra investigación, más que crear un entorno, hemos elaborado dos; uno, que podríamos denominarlo como el propio PLE, y otro, que podríamos considerarlo como un "repositorio de objetos de aprendizaje", en el cual se incorporan en diferentes líneas temáticas los distintos objetos de aprendizaje que hemos elaborado respecto a las mismas.

En la siguiente figura puede observarse el proceso que hemos seguido, para su diseño, producción y evaluación. 


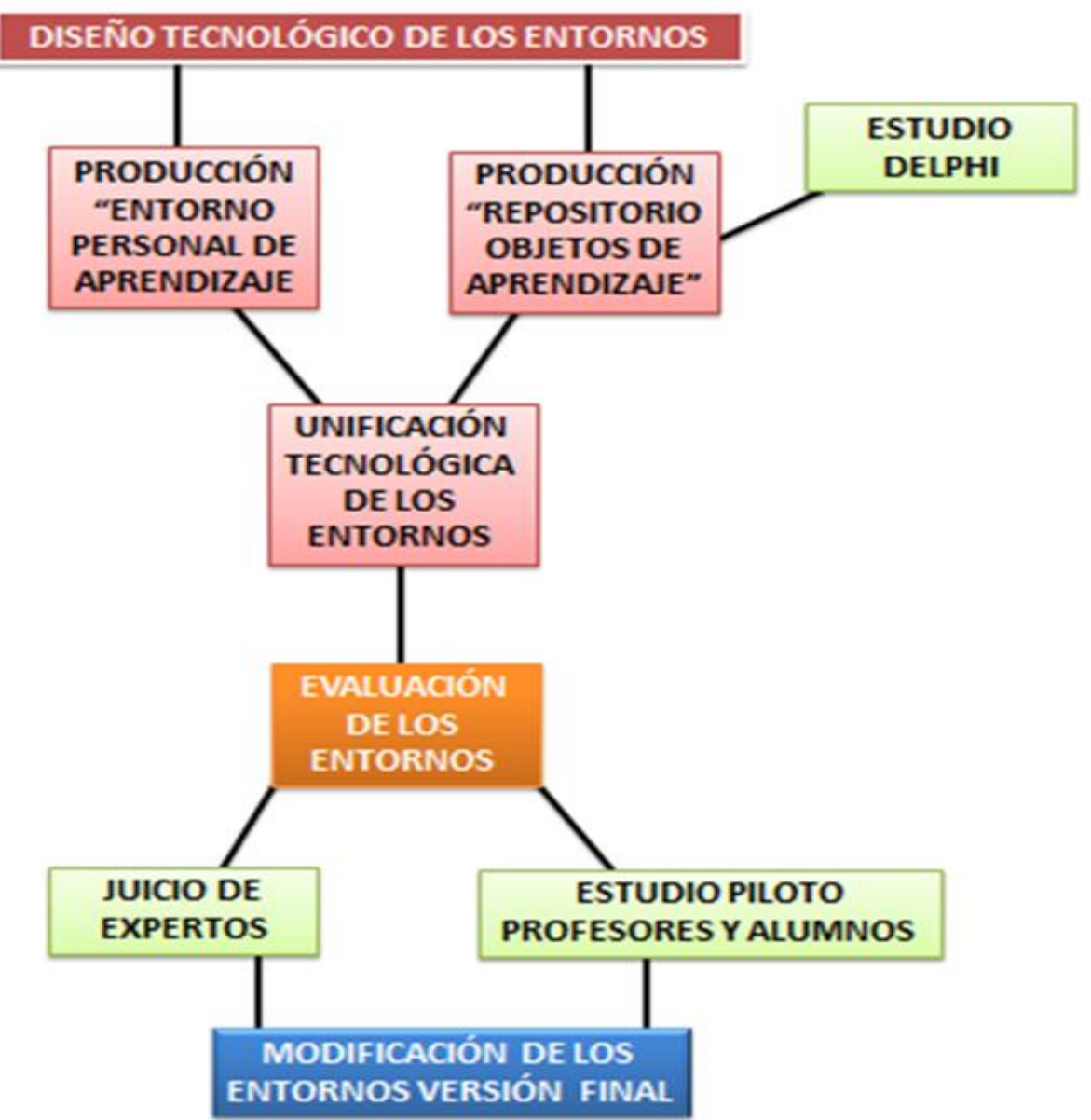

Figura 2. Proceso seguido en el diseño, producción y evaluación del Proyecto Dipro 2.0. Fuente: Elaboración propia.

Como se puede observar, en primer lugar, se elaboraron los dos entornos tecnológicos, que fueron sometidos a diferentes pruebas de evaluación; en concreto, fueron evaluados a través de una serie de expertos que eran profesores de Tecnología Educativa de diferentes Universidades españolas y latinoamericanas, profesores de diferentes universidades españolas a los cuales se le presentaron los entornos en distintas Jornadas de difusión, y mediante los alumnos a través de un estudio piloto llevado a cabo con alumnos que estudiaban contenidos relacionados con la aplicación educativa de las "Tecnologías de la Información y Comunicación” en las Universidades de Sevilla, Córdoba y Sevilla.

Por lo que se refiere al primero de los entornos, el que podríamos considerar como un PLE, en la figura que presentamos a continuación podemos observar su imagen. La dirección web desde la que se puede observar es: http://tecnologiaedu.us.es/portal/ 


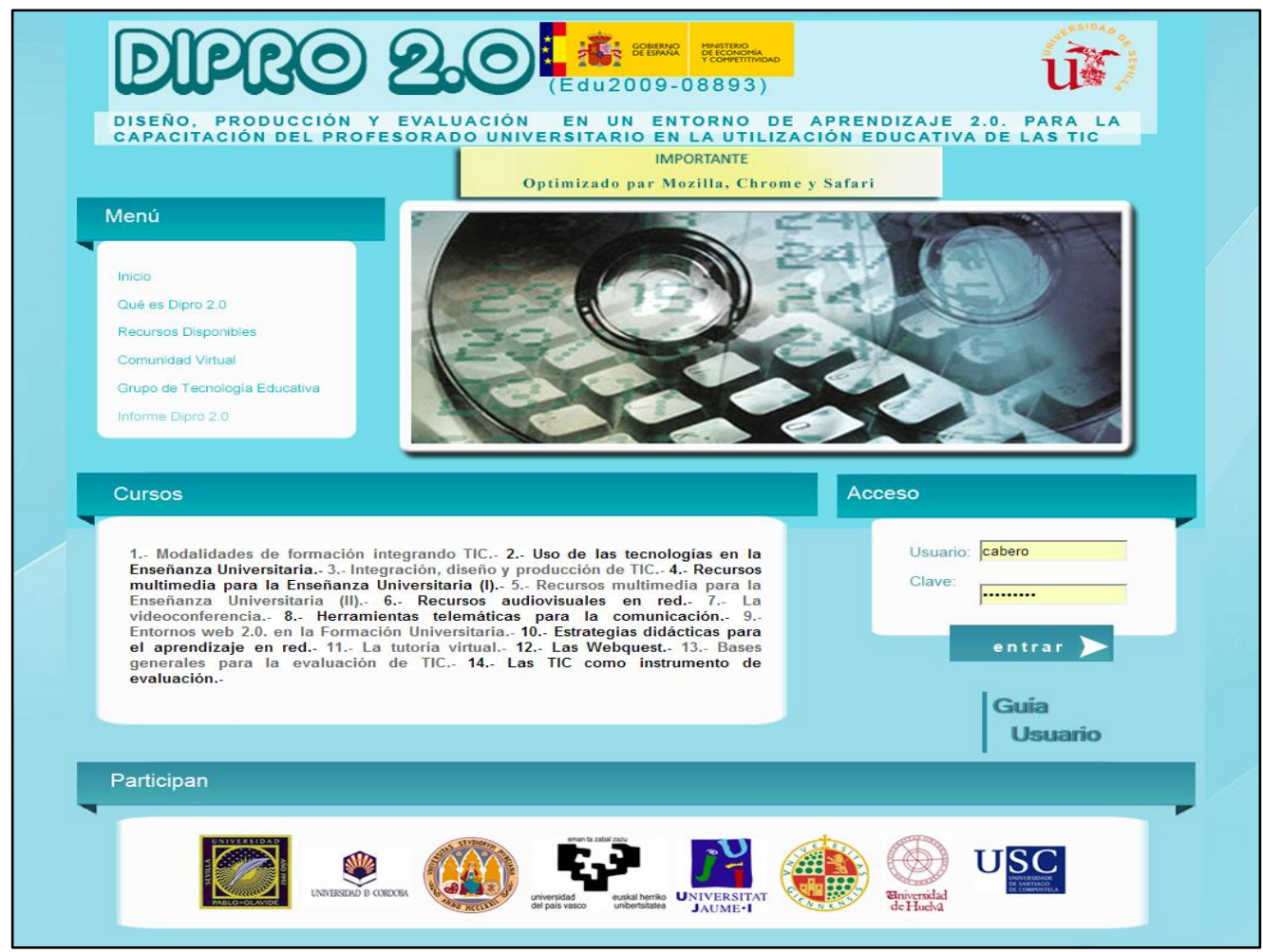

Figura 3. Entrada PLE del proyecto Dipro 2.0.Fuente: http://tecnologiaedu.us.es/portal/

Indicar que se ha pretendido configurar un entorno que pudiera poner a disposición de los alumnos por una parte, un LMS, y por otra, las herramientas más usuales que los estudiantes pueden movilizar para la construcción de un PLE; en definitiva, lo que hemos pretendido construir se podría denominar como un "ePLE" o "Plearning"; es decir, crear un entorno que permitiera utilizar las ventajas que pueden ofrecernos los dos sistemas de formación virtual. En la siguiente figura pueden observarse las dos zonas con las cuales se encuentra el usuario una vez dentro del entorno.

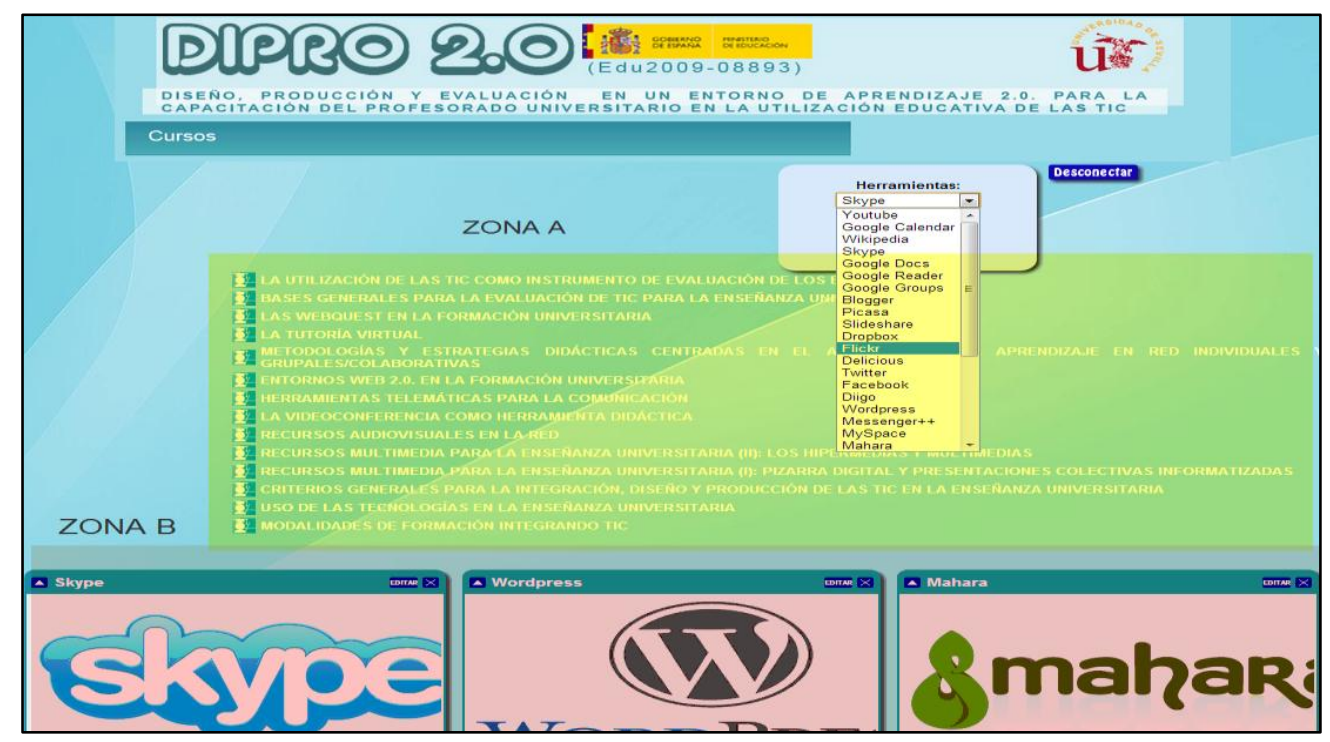

Figura 4. Ubicación del LMS (zona A) y de las herramientas web 2.0 (zona B) en el PLE del entorno Dipro 2.0. Fuente: Entorno Personal de Aprendizaje Dipro 2.0 (http://tecnologiaedu.us.es/portal/) 
Para su construcción se han utilizado diferentes tipos de tecnologías, tal como se recoge en el trabajo de Alfonso, Gallego y Sánchez (2013), señalando que "El producto resultante es un conjunto de módulos con funcionalidad específica y completa que se acoplan entre sí. A su vez, estos módulos se conectan a una plataforma de e-learning mediante la utilización de servicios de base descritos en una capa estándar que funciona como gateway. Un OKI Gateway es un software que traduce las peticiones y solicitudes entre los módulos y las API de la plataforma." (Alfonso, Gallego y Sánchez $(2013,191)$.

Para su realización fue fundamental apoyarse en las siguientes tecnologías: para el LMS la plataforma Moodle como LMS (aunque podría ser otro como Sakai o herramientas propietarias) y un servicio basado en el estándar OSID (Open Service Inerface Definitions) de OKI (The Open Knowledge Iniative), que nos permitía la creación de una arquitectura tipo SOA ("Service Oriented Architecture), y con ella la identificación de una serie de "widgest" que nos permitiría incorporar al entorno de forma rápida y fácil diferentes herramientas de las web 2.0 (Youtube, Google calendar, Skype, Google docs, Blogger, Picasa,....). En el momento el cual se escribe el artículo hay 31 disponibles.

Para la selección de los "widgest", el procedimiento que se siguió fue triple: en primer lugar, se elaboró una primera lista por parte de los miembros del equipo de investigación, lista que fue ampliada por los expertos que evaluaron el entorno, y finalmente fue completada por la propuesta que realiza anualmente por el "Centre for Learning \& Performance Technologies" respecto a las 100 tecnologías de la web 2.0 más utilizadas por los profesores (http://c4lpt.co.uk/).

Por lo que se refiere al segundo entorno, el que es considerado como "repositorio de objetos de aprendizaje", señalar que la diferencia del anterior es fundamentalmente que éste es de acceso libre.

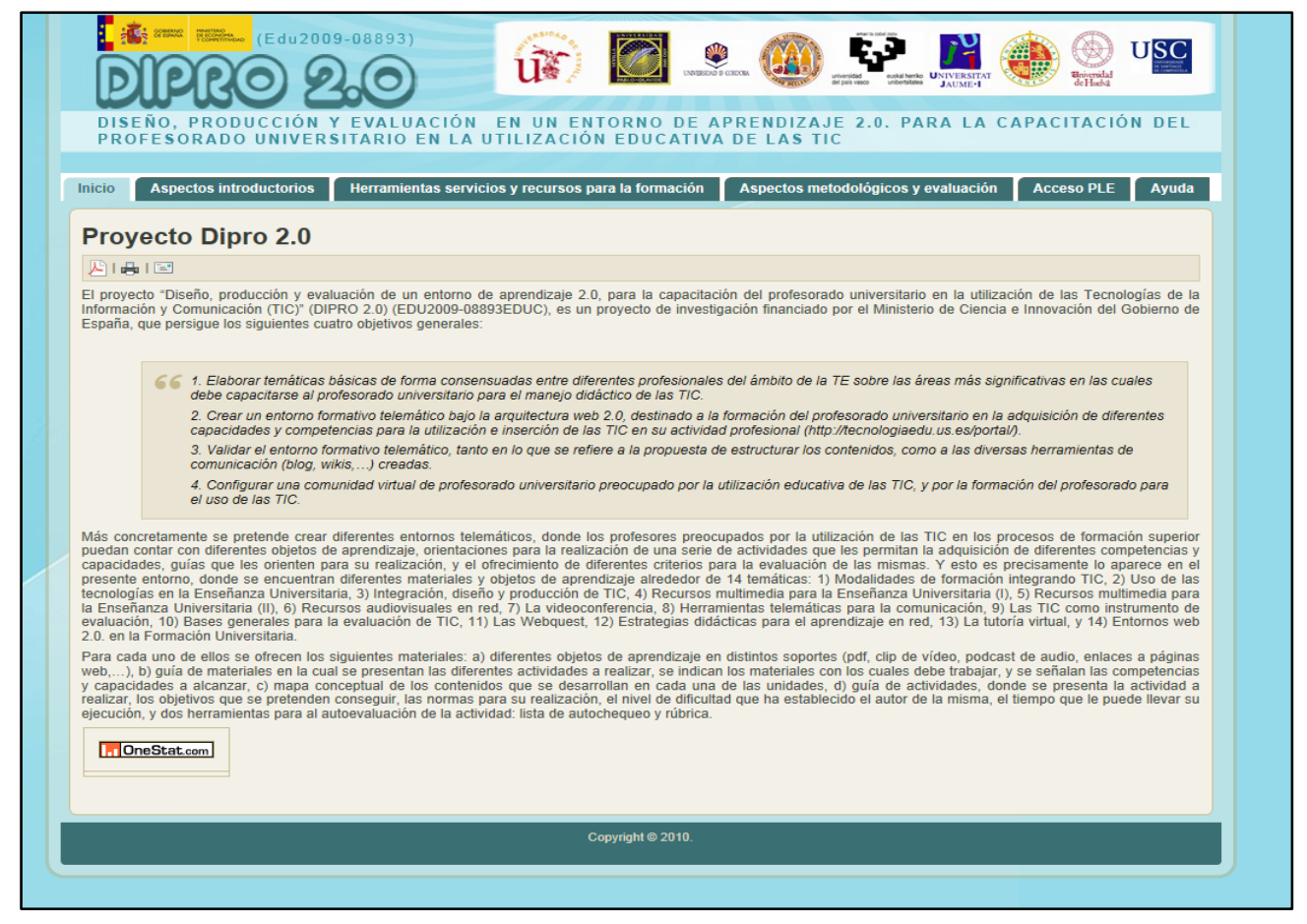

Figura 5. Entrada al repositorio de objetos de aprendizaje del Proyecto Dipro 2.0. Fuente: http://tecnologiaedu.us.es/dipro2/. 
El entorno se organiza alrededor de 14 temáticas, elaboradas a partir de un estudio Delphi con experto realizado a dos vueltas:

1. Modalidades de formación integrando TIC: enseñanza presencial, e-learning, blearning y m-learning.

2. Uso de las tecnologías en la enseñanza universitaria.

3. Criterios generales para la integración, el diseño y la producción de las TIC en la enseñanza universitaria.

4. Recursos multimedia para la enseñanza universitaria (I): pizarra digital y presentaciones colectivas informatizadas.

5. Recursos multimedia para la enseñanza universitaria (II): los hipermedias y multimedias.

6. Recursos audiovisuales en la red.

7. Herramientas telemáticas para la comunicación.

8. La videoconferencia como herramienta didáctica.

9. Entornos web 2.0 en la formación universitaria - herramientas web 2.0.

10. Metodologías y estrategias didácticas centradas en el alumno para el aprendizaje en red individuales y grupales/colaborativas.

11. La tutoría virtual.

12. La Webquest y la formación universitaria.

13. Bases generales para la evaluación de TIC para la enseñanza universitaria.

14. La utilización de las TIC como instrumento de evaluación de los estudiantes.

Es de señalar que, por lo que se refiere al diseño de materiales, se ha elaborado una propuesta de diseño centrado (Cabero, 2012) no en la presentación de contenidos, sino fundamentalmente en la realización de e-actividades por parte de los estudiantes, al entender que las mismas son una variable significativa para que el alumno aprenda en los en los entornos de formación virtual (Cabero y Román, 2006; Sancho y Borges, 2011).

Los materiales se diseñaron de manera que incorporarasen diferentes tipos de elementos; en concreto:

- Guía de materiales.

- Guía de actividades.

- Análisis de las e-actividades en función de la taxonomía de la era digital de Bloom (Chursches, 2009)

- Mapa conceptual de la unidad.

- Y relación de objetos de aprendizaje seleccionados. Objetos de aprendizaje que se presentan en diferentes formatos: pdf, cursos completos, clip de vídeos, multimedias,...). 


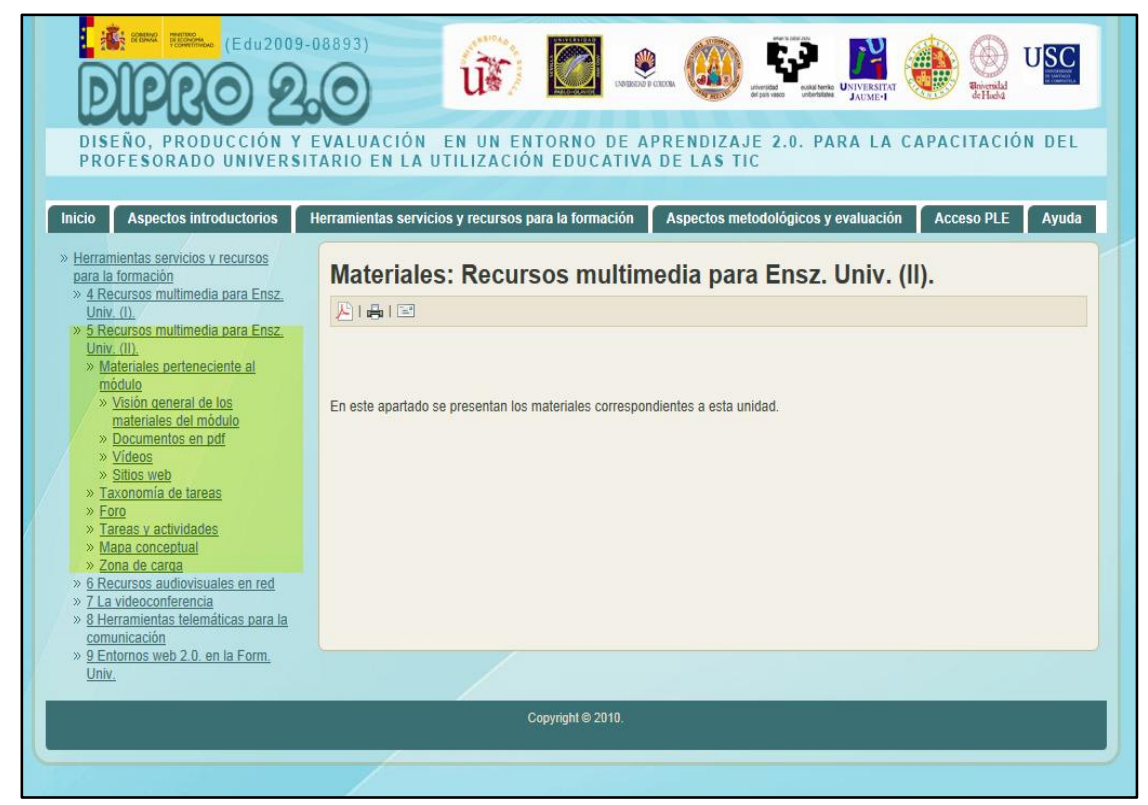

Figura 6. Estructura de los materiales incorporados en cada unidad. Fuente: http://tecnologiaedu.us.es/dipro2/.

Es conveniente destacar que en cada uno de los materiales se incorporan una serie de características, como son: que los materiales pueden ser observados dentro del entorno creado, o descargárselo por el usuario en SCORM, para poder observarlo en su entorno o puntuarlo para que otra persona que desee utilizarlo cuente con un elemento de referencia para su posible utilización.

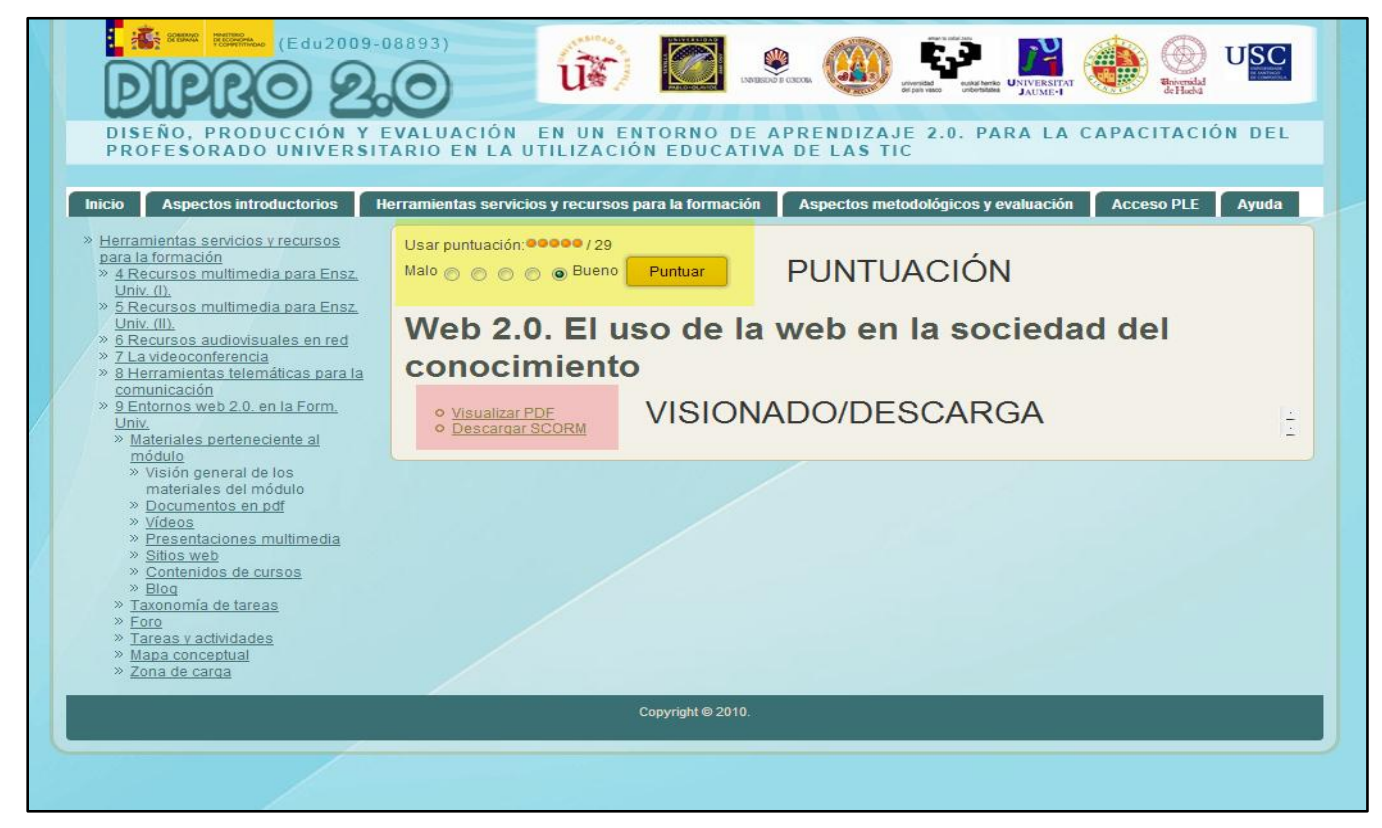

Figura 7. Características de los materiales. Fuente: http://tecnologiaedu.us.es/dipro2/.

Para la elaboración del entorno denominado como repositorio de objetos de aprendizaje, se utilizó el programa gestor de contenidos Joomla 2.5 .

En cuanto al sistema hardware que soporta la estructura del proyecto, hay que decir que se apoya en los siguientes dispositivos, con las siguientes características: 
- Sistema operativo: Windows Server 2008 R2 Standard

- Procesador: Intel Xeon 2Ghz

- Memoria: 4GB

- Software: Apache 2.2, MySQL 5.0, PHP 5.2.

\section{UNAS REFERENCIAS FINALES.}

La evaluación del entorno la hemos llevado a cabo mediante tres procedimientos: evaluación por expertos, evaluación por profesores y evaluación por los estudiantes. Aunque aquí no vamos a presentar los resultados que hemos encontrado, si queremos señalar que tanto a nivel técnico, como estético y en lo referido a su posible incorporación a prácticas educativas, los dos entornos fueron valorados muy positivamente por parte de los tres colectivos.

Por otra parte, llevamos a cabo una experiencia con estudiantes de estudios de magisterio de las Universidades de Sevilla, País Vasco y Córdoba, y en las mismas los alumnos modificaron significativamente los conocimientos que inicialmente tenían hacia la temática de Internet, que fueron los contenidos que habíamos seleccionado para ello.

Los objetivos que se planificaron, así como los resultados obtenidos a través de las diferentes evaluaciones realizadas en el proyecto, podemos apuntar que han sido en su mayoría positivos, es decir, que tanto los aspectos tecnológicos como didácticos a la hora de la construcción y aplicación práctica del PLE han dado resultados satisfactorios, tanto por parte del profesorado como por parte del alumnado. Quizás se puede apuntar una pequeña reflexión final con respecto a la temática que estamos abordando en el presente artículo, y es que en muchas ocasiones seguimos encontrando un discurso confuso en torno a la aplicación didáctica de los PLE, resultando complicado - y a veces imposibleacudir a un modelo de diseño instruccional existente en torno al mismo. De ahí que el desarrollo de experiencias, estudios e investigaciones abordando diferentes variables con respecto a los PLE siga siendo necesario para poder incorporar, de manera adecuada, estas herramientas pedagógicas a nuestra práctica educativa como docentes.

\section{REFERENCIAS}

Adell, J. Y castañeda, L. (2010). Los Entornos Personales de Aprendizaje (PLEs): una nueva manera de entender al aprendizaje. En R. Roig \& M. Fiorucci (Eds), Claves para la investigación en innovación y calidad educativas. La integración de las Tecnologías de la Información y la Comunicación y la Interculturalidad en las aulas. Alcoy: MARFIL-Roma TRE Universita degli estudi.19-30.

Adell, J. (2013). Entornos personales de aprendizaje (PLE). En I. AGUADED, \& J. CABERO (Coords.), Tecnologías y medios para la educación en la e-sociedad. Madrid: Alianza, 271-288.

Amine, M. (2009). PLE $-P K N$. Recuperado el 12/01/2010 de: http://mohamedaminechatti.blogspot.com/2009/04/ple-pkn.html 
Ampudia, V. y trinidad, L. (2012). Entornos personales de aprendizaje: ¿final o futuro de los EVA?. Reencuentro, 63, 32-39.

Attwell, G. (2007). The Personal Learning Environments - the future of eLearning? eLearning Papers, 2, 1.

Banyard, PH. y underwood, J. (2008). Understanding the learning space. eLearning Papers, 9, Recuperado el 3/01(2012 de: www.elearningpapers.eu

Barroso, J., cabero, J. y vázquez, A. (2012). Formación desde la perspectiva de los entornos personales de aprendizaje. Apertura, Recuperado el 18/03/2012 de: http://www.udgvirtual.udg.mx/apertura/index.php/apertura3/article/view/209

Bauman, Z. (2007). Los retos de la educación en la modernidad líquida. Barcelona: Gedisa.

Cabero, J. (2012). Tendencias para el aprendizaje digital: de los contenidos cerrados al diseño de materiales centrado en las actividades. El Proyecto Dipro 2.0. RED. Revista de Educación a Distancia, 32, Recuperado el 18/03/2012 de: http://www.um.es/ead/red/32.

Cabero, J. y otros (2011). Creación de un entorno personal para el aprendizaje: desarrollo de una experiencia. EDUTEC, Revista Electrónica de Tecnología Educativa, 38. Recuperado el 20/01/2012 Recuperado de: http://edutec.rediris.es/Revelec2/Revelec38/creacion_entorno_personal_aprendiza je desarrollo experiencia.html

Cabero, J. Y román, P. (2006). E-actividades. Un referente básico para la formación en Internet. Sevilla: Eduforma.

Casquero, O. y otros (2010). ¡PLE Network: an integrated eLearning 2.0 architecture from a university's perspective. Interactive Learning Environment, vol. 18, núm. 3, 293-308.

Castañeda, L. Y adell, J. (eds.). (2013). Entornos personales de aprendizaje: claves para el ecosistema educativo en red. Alcoy: Marfil.

Cedefop (2008). Terminology of European Education and traigning policy. Luxemburgo: Publications Office.

Dabbagh, N. Y kitsantas, A. (2012). Personal Learning Environments, social media, and selfregulated learning: a natural formula for connecting formal and informal learning. Internet and Higher Education, 15, 3-8.

Dabbagh, N., Y reo, R. (2011). Impact of Web 2.0 on higher education. En D. W. Surry, T. Stefurak, y R. Gray (Eds.), Technology integration in higher education: Social and organizational aspects. Hershey, PA: IGI Global, 174-187.

Downes, S. (2007). Learning networks in practice. Emerging Technologies for Learning, 2, 19-27.

Durall, E y otros (2012). Perspectivas tecnológicas: educación superior en Iberoamérica 2012-2017. Austin, Texas: The New Media Consortium. 
Fiedler, S. Y pata, K. (2009). Distributed learning environments and social software: In search for a framework of design. En S. Hatzipanagos y S. Warburton (Eds.). Social software \& developing community ontologies. Hershey, PA: IGI Global, 145-158.

Henri, F. y otros (2008). Understanding PLE as an Essential Component of the Learning Process. Proc. of ED-Media, AACE, Chesapeake, 3766-3770.

Infante, A, gallego, O. Y sánchez, A (2013). Los gadgests en las plataformas de teleformación: el caso del proyecto Dipro 2.0. Pixel-Bit. Revista de Medios y educación, 42, 183-194.

Johnson, L., y otros (2011). The NMC Horizon Report: 2011 K-12 Edition. Austin, Texas: The New Media Consortium.

Kitsantas, A., Y DABBAGH, N. (2010). Learning to learn with Integrative Learning Technologies (ILT): A practical guide for academic success. Greenwich, CT: Information Age Publishing.

Llorente, M.C (2013). Aprendizaje autorregulado y PLE. Edmetic. Revista de Educación Mediática y TIC, no 1, Vol. 2, 63-79.

Marín, V. (2011). Implicaciones pedagógicas de IPLE: ambientes de aprendizaje personales e institucionales (Proyecto fin de Master no publicado). Universidad de las Islas Baleares: España.

Mott, J. (2010). Envisioning the Post-LMS Era: The Open Learning Network. Educause Quartely, 33 (1). Recuperado el 12/01/2010 de: http://www.educause.edu/EDUCAUSE+Quarterly/ EDUCAUSE QuarterlyMagazineVolum/ EnvsioningthePostLMSEraTheOpe/199389.

Reig, D. (2009). Entornos personales de aprendizaje. Recuperado el 26/08/2012 de: http://www.slideshare.net/dreig/ple-1340811

Rodríguez, M. (2013). Una experiencia de creación de un entorno personal de aprendizaje. Proyecto Dipro 2.0. Edmetic. Revista de educación Mediática y TIC, 2, 1, 7-22.

Sancho, t. Y borges, f. (2011). El aprendizaje en un entorno virtual y su protagonista, el estudiante virtual. En Gros, B. (ed.), Evolución y reto de la educación virtual. Construyendo el e-learning del siglo XXI. Barcelona, UOC., 27-49.

Sclater, N. (2008). Web 2.0, Personal Learning Environments, and the future of leanring management systems. Educause, 13, 1-13.

Starkey, L. (2011). Evaluating learning in the 21st century: a digital age learning matrix. Technology, Pedagogy and Education, 20, 1, 19-39.

Taraghi, B. y otros (2009). Personal Learning Environment - a Conceptual Study, Conference ICL2009, Recuperado el 26/06/2012 de: http://es.scribd.com/doc/20156291/Personal-Learning-Environment-A-ConceptualStudy 
Uztarrroz, J. (2010). Creación y gestión de un PLE (Personal Learniong Environment Entorno Personal de Aprendizaje), Recuperado el 3/06/2012 de: https://docs.google.com/file/d/OB2UkCOAzGKAgNzMwMWVkZWUtYTcyZCOOYmMw LTIIMzQtNzI4NTAONjcxYTU2/edit?pli=1

Väljataga, T., Pata, K., y Tammets, K. (2001). Considering students' perspective on personal and distributed learning environments. En M. J. W. Lee, \& C. McLoughlin (eds.), Web 2.0-based e-Learning: Applying social informatics for tertiary teaching. Hershey, PA: IGI Global, 85-107.

Wilson, S. (2008). Patterns of personal learning environments. Interactive Learning Environments, 16, 1, 17-34.

\section{Para citar este artículo:}

Cabero, J. Creación de entornos personales de aprendizaje como recurso para la formación. El proyecto Dipro 2.0 EDUTEC, Revista Electrónica de Tecnología Educativa, 47. Recuperado el $\mathrm{dd} / \mathrm{mm} /$ aa de http://edutec.rediris.es/Revelec2/Revelec47/n47 Cabero.html 